Please do not remove this page

RMIT

UNIVERSITY

\title{
Fractal theory based non-linear analysis of sEMG
}

Poosapadi Arjunan, Sridhar; Kumar, Dinesh

https://researchrepository.rmit.edu.au/esploro/outputs/9921863505701341/filesAndLinks?institution=61RMIT_INST\&index=null

Poosapadi Arjunan, S., \& Kumar, D. (2007). Fractal theory based non-linear analysis of sEMG. Third International Conference on Intelligent Sensors, Sensor Networks and Information Processing (ISSNIP), 545-548. https://doi.org/10.1109/ISSNIP.2007.4496901

Published Version: https://doi.org/10.1109/ISSNIP.2007.4496901

Repository homepage: https://researchrepository.rmit.edu.au (C) 2007 IEEE

Downloaded On 2023/04/26 14:31:04 +1000

Please do not remove this page 


\title{
Fractal theory based Non-linear analysis of sEMG
}

\author{
* Sridhar P Arjunan, Dinesh K Kumar \\ School of Electrical and Computer Engineering, RMIT University \\ GPO Box 2476V, Melbourne, Victoria 3001, Australia \\ sridhar_arjunan@ieee.org
}

\begin{abstract}
This research examines the use of fractal theory to study the properties of surface electromyogram (sEMG). The paper reports identifying a new fractal feature, maximum fractal length (MFL) that, along with fractal dimensions, has been found to be useful in modelling the muscle activity. Experimental results demonstrate that the combination of fractal dimension and maximum fractal length of sEMG recordings is suitable for characterising the activation of subtle gestures. The log-log plots demonstrate the presence of patterns of these features for each of the hand gesture that can be related to the muscle anatomy. The results indicate that there is small inter-experimental variation but there are large inter-subject variations. This inter-subject variation may be attributable to anatomical differences for the different subjects. The possible applications of this research include use of classifying $s E M G$ for controlling machines and computers.
\end{abstract}

\section{INTRODUCTION}

Surface Electromyogram (sEMG) is a surface recording of the underlying muscle activity. The strength of sEMG is a good measure of the strength of contraction of the muscle, and can be related to the movement and posture of the corresponding part of the body. SEMG reflects functional state of muscle fibres [4]. sEMG is a complex and non-stationary signal. The mechanism of sEMG is very complicated that every motor neuron has its own discharge threshold and recruiting level, which defines a muscle system as a complex nonlinear system [11].

The methods used in sEMG analysis are linear in nature. The uses of linear methods are that they are often quite simple to implement and the interpretation of the results is more or less straightforward. In many cases the physiological systems underlying the sEMG are highly complex and, hence it is reasonable to assume that the mechanism generating the sEMG is nonlinear in nature. The properties describing nonlinear signals can be estimated by calculating nonlinear measures such as entropies, correlation and fractal dimensions, and selfcorrelation [8]. The use of nonlinear methods has increased substantially during the past decade and will be useful in characterizing sEMG.

Examples of new approaches in nonlinear methods have been proposed in literature for characterization of sEMG. They are spectral distribution i.e. Logarithmic representation of EMG spectrum, Poisson representation of EMG spectrum and the method that examines the 'complexity' of raw EMG i.e., Fractal dimension of sEMG. Out of these approaches, fractal dimension of sEMG has been found sensitive to magnitude and rate of force of generation [14].

The non-periodic, turbulent property of the sEMG signal can be measured using fractals, by calculating fractal dimension. The fractal dimension is introduced as the index for describing the irregularity of this type of time series [1]. Recent works has been done on Fractal analysis of EMG signal. Gitter et. al [3] demonstrated that the fractal characteristics of EMG signal with a dimension is highly correlated with muscle force. Gupta et. al [2] reported that the fractal dimension can be used to characterise the EMG signal. Hu et al. [7] distinguished two different patterns of ASEMG signals by their fractal dimension from the filtered ASEMG signals. These studies demonstrate that fractal dimension can be used as a measure in characterising the EMG signal.

However, the characterisation using fractal dimension is highly limited. There is need to determine fractal features of sEMG that can be related to different aspects of muscle contraction such as levels of muscle contraction, size of muscles and depth of the active muscle. This may be useful in studying the sEMG model for subtle actions. In this paper, the authors report the use of fractal dimension and maximum fractal length of the sEMG signal from different recordings as features to study the model of sEMG for classification of different hand motions. The paper reports the results for the performance of these features for measure of muscle activity with respect to anatomical details of muscle.

\section{THEORY}

Many natural objects exhibit the property that as one views the object at greater manifestations, more and more similar structures are revealed. Fractals exhibit this self-similar property [5]. The source of sEMG is a set of similar action potentials originating from different locations in the muscles. Because of the self - similarity of the action potentials that are the source of the sEMG recordings over a range of scales, sEMG is expected to have fractals properties.

\section{A. Self-similarity}

Self-similarity is the scale invariance of a process and is a distinctive feature of most fractals. Self-similar processes are the ones in which a small portion of the process resembles a larger section when suitably magnified. Self-similarity, in a 
strict sense, means that the statistical properties of a stochastic process do not change for all aggregation levels of the stochastic process. The stochastic process 'looks the same' irrespective of any magnification of the process. Exactly selfsimilar fractal objects are identical regardless of the scale or magnification at which they are viewed [12]. For the signal or process, $y(k)$ to be self-similar the variance of the aggregated process decays slowly with $m$ and this selfsimilarity is measurable by $H$ that is,

$$
\begin{gathered}
\operatorname{Var}\left(y^{(m)}(k)\right) \approx m^{-\beta} \\
\text { with } 0<\beta<1 \text { and } H=1-\beta / 2,
\end{gathered}
$$

where $H$ expresses the degree of self similarity; large values indicate stronger self-similarity.

\section{B. Fractal dimension}

Fractals refer to objects or signal patterns that have fractional dimension. These objects exhibit self-similarity. This defines that the objects or patterns on any level of magnification will yield a structure that resembles the larger structure in complexity. The measured property of the fractal process is scale dependant and has self-similar variations in different time scales. Fractal dimension (FD) is a measure of the fractal properties of any structure.

Biosignals such as sEMG are a result of the summation of identical motor units that travel through tissues and undergo spectral and magnitude compression. Researchers have studied fractal of sEMG to characterize normal and pathological signals. Anmuth et al. [6] determined that there was a small change of the fractal dimension of the EMG signal and this was linearly related to the activation of the muscle measured as a fraction of maximum voluntary contraction. They also observed a linear relationship between the fractal dimension and the flexion-extension speeds and load. Gitter et al determined that fractal dimension can be used to quantify the complexity of motor unit recruitment patterns [3]. Fractal dimension is a measure of the source properties and hence the authors propose to use it as a measure of the overall muscle properties. The fractal dimension is estimated by measuring the change in the length of the curve with the change in the measurement scale. Muscle dimensions and complexity are inherent properties of the muscle. This complexity may change with the change in shape and contraction of the muscle. While high level of contraction or muscle stretch would also have an impact on FD [2], there would not be significant variations of FD with small changes of muscle contraction.

While FD of the signal would remain the same when there are small changes in the muscle activity, the overall length of the signal would change when there are more MUAPs. The authors propose to use the overall length of the signal as a measure of the muscle activity. Based on the experimental results, this paper reports that while FD may remain same for a muscle even though there is a small change in muscle contraction, maximum fractal length (MFL) measured as the absolute value of the length of the signal at the lowest scale follows the small changes in muscle contraction.
TABle 1: Placement of Electrodes over the SKIN of The forearm

\begin{tabular}{|l||l|}
\hline Channel & Muscle \\
\hline 1 & Brachioradialis \\
\hline 2 & Flexor Carpi radialis (FCR) \\
\hline 3 & Flexor Carpi Ulnaris (FCU) \\
\hline 4 & $\begin{array}{l}\text { Flexor digitorum superficialis } \\
\text { (FDS) }\end{array}$ \\
\hline
\end{tabular}

An obvious application of these properties of sEMG is the identification of small changes in muscle activity in the presence of multiple active muscles. This paper also reports experiments conducted to verify this by classifying FD and MFL of sEMG recorded from the forearm identify small hand and wrist gestures.

\section{Methodology}

Experiments were conducted to determine fractal dimension and maximum fractal length for the study of sEMG model and for further classification. In order to determine the level of muscle activity, subtle hand gestures were chosen as experiments. This could be helpful in our study to determine FD and MFL at smallest level of gestures.

\section{A. SEMG Recording and Experiments}

Experiments were approved by University ethics committee to conduct experiments on human subjects and acquire sEMG using surface electrodes. Six male participants with no history of myo or neuro-pathology were chosen for experiments. For the data acquisition a sEMG acquisition system by Delsys (Boston, MA, USA) was used. Four electrode channels were placed over four different muscles [4], [9] as follows (refer Table.1): Brachioradialis Flexor Carpi radialis (FCR), Flexor Carpi Ulnaris (FCU), Flexor digitorum superficialis (FDS).

Each channel is a set of two differential electrodes with a fixed inter-electrode distance of $10 \mathrm{~mm}$ and a gain of 1000 . Before placing the electrodes subject's skin was prepared by lightly abrading with skin exfoliate to remove dead skin that helps in reducing the skin impedance to less than $60 \mathrm{k} \Omega$. Skin was also cleaned with $70 \% \mathrm{v} / \mathrm{v}$ alcohol swab to remove any oil or dust on the skin surface. The following four different hand gestures were used as protocol to record sEMG from the participants: Wrist flexion, Index and Middle finger Flexion, Wrist flexion (towards little finger in horizontal plane), all finger flexion.

\section{B. Data Analysis}

As a first step of data analysis, fractal dimension and maximum fractal length for sEMG recordings of hand gestures, (refer Fig.1) were computed. These features were analyzed in relation to the level of muscle activity and its activity pattern with respect to the muscle anatomy. The performance of the feature set for classification against the corresponding hand gestures. At the second instance, the fractal property of recorded sEMG signal was analyzed by determining fractal dimension. Fractal dimension was calculated using the following procedure reported by Higuchi [1] for irregular time 


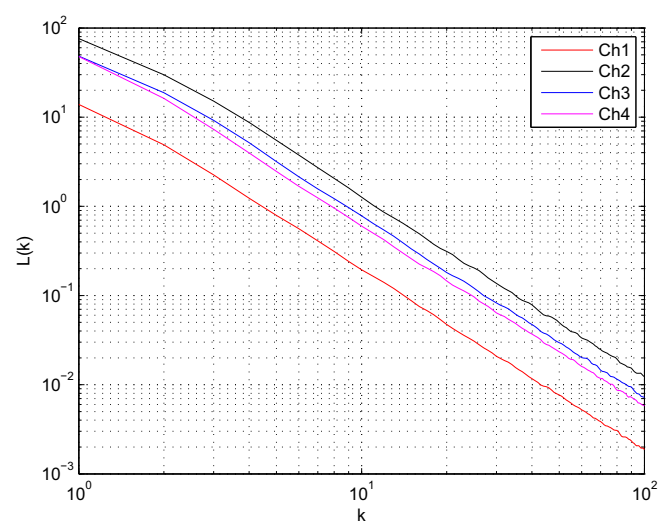

(a)

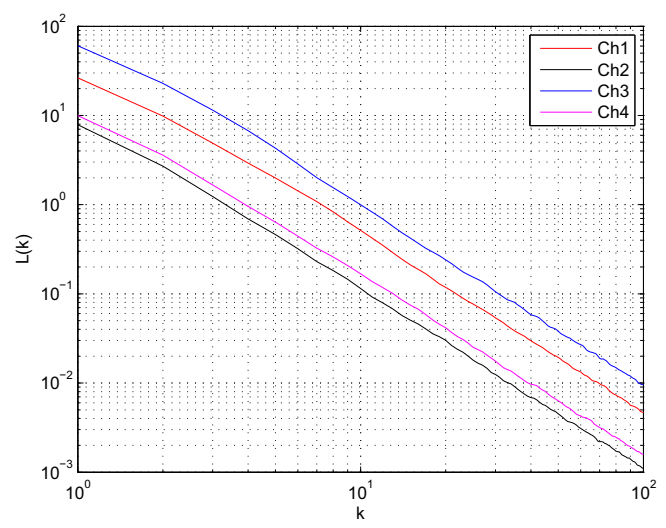

(b)

Fig. 1: Example of Logarithmic plot of the curve length $\langle L(k)\rangle$ and $k$ for the four channel recorded sEMG signal during different hand gestures a) Wrist flexion, b)Wrist flexion (towards little finger in horizontal plane)

series. This procedure yields a more accurate estimation of fractal dimension [13].

- Defining the length of the curve, $X_{k}^{m}$, as in (1)

$$
L_{m}(k)=\frac{\left(\sum_{i=1}^{\left[\frac{N-m}{k}\right]}|X(m+i k)-X(m+(i-1) \cdot k)|\right) \frac{N-1}{\left[\frac{N-m}{k}\right] \cdot k}}{k}
$$

The length of the curve for the time interval $k,\langle L(k)\rangle$ is defined as the average value over $k$ sets of $L_{m}(k)$. If $\langle L(k)\rangle \propto$ $k^{-D}$, then the curve is fractal with the dimension - D.

From the logarithmic plot, the Maximum Fractal Length i.e., the value of the length of the curve at the lower scale which represents the originating data point for the each sEMG signal for the respective hand motions was determined. This MFL represents the level of muscle activity for the particular gestures. The logarithmic plot of the modified sEMG signal for each of the four channels for all hand gestures was plotted. Each individual plot contains the curve for four channels for the each of the hand gesture. This is shown in Fig.1 and Fig.2. This gives different pattern of muscle activation for different gestures. These patterns were related to the muscle anatomy using MFL as a measure of activation. The MFL and Fractal

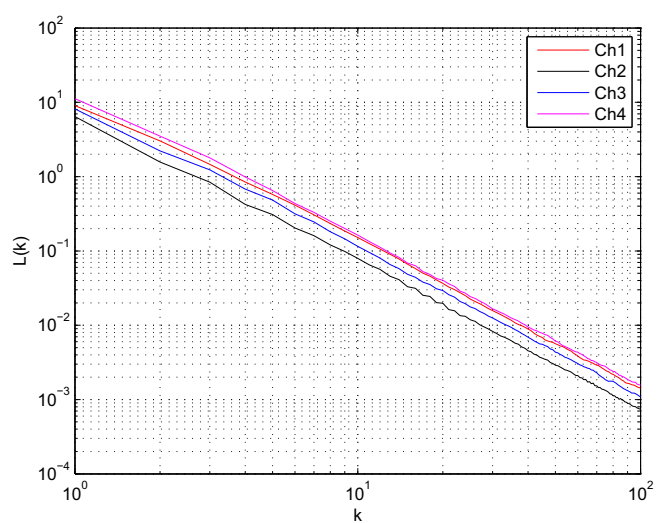

(a)

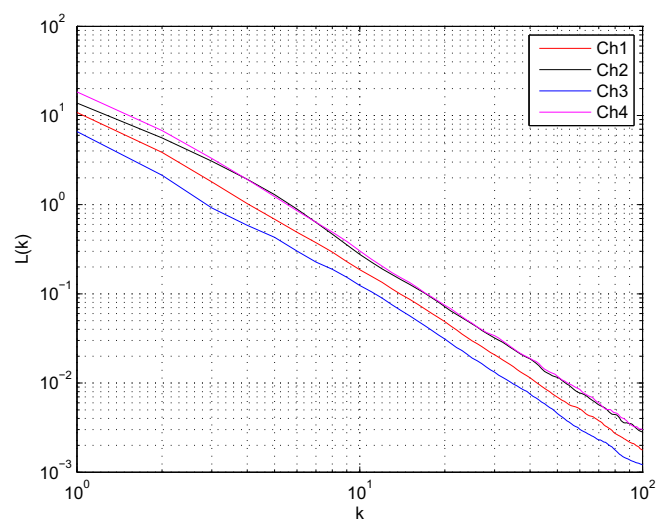

(b)

Fig. 2: Example of Logarithmic plot of the curve length $\langle L(k)\rangle$ and $k$ for the four channel recorded sEMG signal during different hand gestures a) Index and Middle finger flexion, b)All fingers flexion

dimension for each sEMG Channel was determined from the logarithmic plot and tabulated.

\section{RESULTS AND Discussion}

The feature set from the four hand gestures were used to classify the gestures with respect to the muscle activity pattern. The logarithmic plot of the signal length and scale for the four different hand gestures were plotted as shown in Fig.1 and Fig.2. From the example plot, it is observed that there is a clear pattern for each of the gestures. Each sEMG from the different channel for a particular hand gesture has a specific pattern observable based on the slope of the line and point of intersection on the y-axis. The point of intersection with the $y$-axis is the point of lowest scale, and is the maximum fractal length (MFL).

From the results of all the subjects, there are some common observations: For Wrist Flexion gesture, Channel 2 has higher maximum fractal length i.e., level of muscle activity and for Wrist Flexion gesture towards little finger in horizontal plane, Channel 3 has higher MFL which relates to the muscle anatomy [4], [9] (refer Fig.1). While for fingers flexion gestures, Channel 4 has higher maximum fractal length 


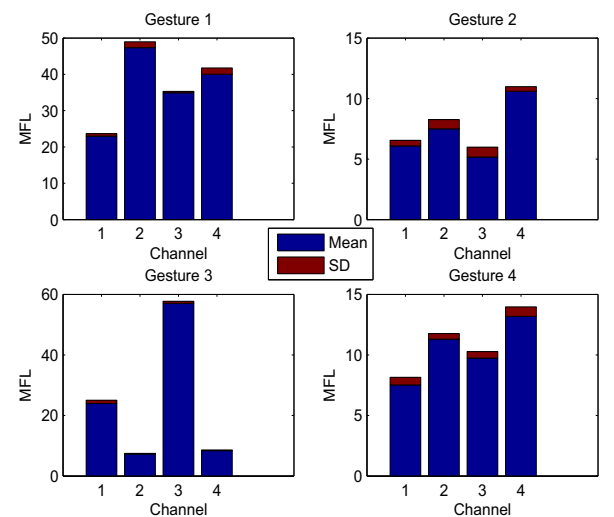

Fig. 3: Bar plot of Maximum Fractal Length for different Gestures from Single subject

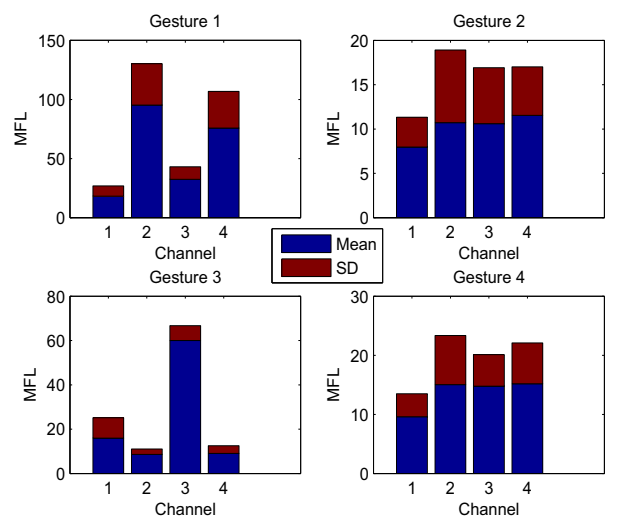

Fig. 4: Bar plot of Maximum Fractal Length for different Gestures from Six subjects

(MFL) at lower scales and as the line decays it overlaps with Channel 2 and Channel 1 at higher scales (refer Fig.2). This may be due overlap of recruitment of motor units for this particular gestures. This can be verified from the bar plots, which explains the mean and standard deviation of MFL for different gestures from different channels for single subject and different subjects shown in Fig. 3 and Fig.4 respectively.

From the plots, it is also observed that there is a significant difference between the MFL values between the different gestures for the different channels. This investigation shows that the patterns are different for different hand gestures and can be used to study the sEMG model for the hand gestures. The feature set of MFL and fractal dimension, calculated from the slope of the line, can be used for classification of these hand gestures.The experimental results were analyzed to determine the inter-subject and intra-subject variations. The fractal dimension for different sEMG signal varied between 1.95 and 2.

From Fig.3, the results indicate that there is very small interexperimental variation for a gesture for each subject, while from Fig.4, it is evident that the standard deviation is much higher, suggesting high inter-subject variation. From Fig.3, it is also observed that there are distinct muscle activation patterns for each of the gestures. While the research work related to ultrasonography is in progress, the authors have observed this to be consistent with ultrasonography.

\section{Conclusion}

This research reports the use of fractal theory to estimate the level and source of muscle activity of different hand gestures for determining the corresponding hand gesture. Fractal dimensions determine the complexity of the sEMG signal and the maximum fractal length represents a measure of muscle activity. This feature is useful for analysis of sEMG patterns when there are multiple active muscles and can be useful in modelling sEMG.

The experiment results have tested the efficacy of MFL and fractal dimension to identify the pattern of sEMG activity corresponding to each hand gesture. The results also demonstrate that there is small inter-experimental variation, while the inter-subject variations are larger. This may be attributable to the differences in anatomy of different subjects. Applications of this include developing more robust model of sEMG, overcoming cross talk issues and identifying muscle activity for applications such as human computer interface.

\section{REFERENCES}

[1] T. Higuchi, "Approach to irregular time series on the basis of the fractal theory," in Physica D, vol. 31, pp. 277-283, 1988.

[2] V. Gupta, S. Suryanarayanan, and N. P. Reddy, "Fractal analysis of surface EMG signals from the biceps," Intl. J. Medical informatics, vol. 45, pp. 185-192, 1997.

[3] J. A. Gitter, and M. J. Czerniecki, "Fractal analysis of electromyographic interference pattern," Journal of Neuroscience Methods, vol. 58, pp. 103$108,1995$.

[4] J. Basmajian, and C. J. De Luca, Muscles Alive: Their Functions Revealed by Electromyography, 5th ed., Baltimore, MD:Williams \& Wilkins, 1985.

[5] B. B. Mandelbrot, Fractals: Form, chance, and dimension, W. H. Freeman and Co., San Francisco, 1977.

[6] C.J. Anmuth, G. Goldberg, and N.H. Mayer, "Fractal dimension of EMG signals recorded with surface electrodes during isometric contractions is linearly correlated with muscle activation," Muscle \& Nerve, vol 17, pp. 953-954, 1994

[7] X. Hu, Z. Wang, and X .Ren, "Classification of surface EMG signal with Fractal dimension," Journal of Zhejiang University Science, vol. 6B, no. 8, pp.844-848, 2005.

[8] P. A. Karjalainen, 'Nonlinear methods in EMG analysis," Biosignal analysis and Medical Imaging Group, University of Kuopio, http://bsamig.uku.fi/research/emg_nonlinear.shtml Last access: March, 2007

[9] Dartmouth Medical School, Ḧuman Anatomy: Muscles of Wrist and Hand," in http://www.dartmouth.edu/\%7Eanatomy/wristhand/muscles/index.html Last access: March, 2007.

[10] Z. Yang, and G. Zhao, "Phase Space Analysis of EMG," ACTA BOIPHYSICA SINICA, vol. 14, no. 2, 1998. In Chinese.

[11] B. Chen, and N. Wang, "Determining EMG Embedding and Fractal Dimensions and its application," in Proceedings of the 22nd Annual EMBS International Conference, Chicago IL USA, pp. 1341-1344, July 23-28, 2000 .

[12] R. Kalden, and S. Ibrahim, "Searching for Self-Similarity in GPRS," in Proceedings of PAM2004, France, April 19-20, 2004.

[13] R. Esteller, G. Vachtsevons, J. Echautz , and B. Litt, "A comparison of Waveform Fractal Dimension Algorithms," IEEE Transactions on Circuit and Systems-I: Fundamental theory and applications, vol. 48, no. 2, pp. 177-183, February 2001

[14] M. A. Nussbaum, "Localized Muscle Fatigue" in Lecture Notes on Advanced Methods in Occupational Biomechanics, http://www.nussbaum.org.vt.edu/courses.htm, Last Access: Aug-2006. 